\title{
REVISIÓN
}

\section{Acumulación de aminoácidos tipo micosporina (MAAs): biosíntesis, fotocontrol y funciones ecofisiológicas}

\author{
Accumulation of mycosporine-like amino acids (MAAs): biosynthesis, photocontrol and \\ ecophysiological functions
}

\author{
NATHALIE KORBEE*, FÉLIX L. FIGUEROA \& JOSÉ AGUILERA
}
Departamento de Ecología, Facultad de Ciencias, Universidad de Málaga, Campus Universitario de Teatinos s/n, 29071, Málaga, España;
*e-mail para correspondencia: nkorbee@uma.es

\begin{abstract}
RESUMEN
Los efectos nocivos que inducen la radiación UV en organismos acuáticos pueden ser aminorados a través de varios mecanismos de fotoprotección entre los que se encuentran la acumulación de sustancias que absorben radiación UV (fotoprotectores) y activación de sistemas antioxidantes. Entre los fotoprotectores descritos se encuentran los aminoácidos tipo micosporina (MAAs). En esta revisión se enfatiza sobre la capacidad fotoprotectora y antioxidante de estas sustancias. La función fotoprotectora de los MAAs puede deducirse de las características fotofísicas de estos compuestos. Es sabido que actúan como pantalla pasiva disipando térmicamente la energía UV absorbida. La acumulación de MAAs se induce tanto por radiación UV (UV-A y UV-B) como por luz azul dentro de la banda de la radiación activa fotosintética (PAR). Por otro lado, hay algunas referencias que indican otras funciones fisiológicas de los MAAs, incluyendo la actividad antioxidante, la regulación osmótica y la reproductora. Siendo los MAAs sustancias nitrogenadas, recientemente se ha comenzado a estudiar la influencia de la disponibilidad de nitrógeno en la acumulación de estos, en combinación con UV y/o alta irradiancia de PAR. El amonio se moviliza hacia los MAAs relativamente rápido, por lo que se ha propuesto también a los MAAs como reservorio de nitrógeno.
\end{abstract}

Palabras clave: aminoácidos tipo micosporina, fotoprotección, nitrógeno, radiación fotosintética, radiación UV.

\begin{abstract}
The negative effects of UV radiation on aquatic organisms can be diminished by several photo-protective mechanisms such as the accumulation of UV absorbing substances (photoprotectors) and the activation of antioxidant systems. The mycosporine-like amino acids (MAAs) are one of the most known photoprotectors. In this review their photoprotective and antioxidant capabilities are described. The UV screening function of MAAs can be inferred from their photophysic characteristics. It is known that MAAs act as passive screen dissipating the UV energy absorbed as thermal form. The accumulation of MAAs is induced by both UV radiation (UV-A and UV-B) and by blue light in the photosynthetic active radiation part of the spectrum (PAR). On the other hand, there are several reports indicating other physiological functions of the MAAs, antioxidant activity, osmotic regulation and reproduction. Since MAAs are nitrogenous compounds, recently the influence of inorganic nitrogen in combination to UV radiation and/or high irradiance of PAR is being investigated. The ammonium is rapidly mobilized to MAAs, and hence they maybe regarded as a nitrogen reservoir.
\end{abstract}

Key words: mycosporine-like amino acids, nitrogen, photoprotection, photosynthetic active radiation (PAR), UV radiation. 


\section{INTRODUCCIÓN}

La radiación solar no llega en su totalidad a la superficie de la Tierra, ciertas longitudes de onda son absorbidas total o parcialmente antes de alcanzarla por moléculas como oxígeno atómico y ozono. La radiación UV-B (280-315 $\mathrm{nm})$ representa solo una pequeña proporción del total de la radiación solar y menos de un $1 \%$ del flujo solar total que alcanza la superficie terrestre, pero se trata de un rango de radiación altamente activa en términos biológicos, ya que los fotones de estas longitudes de onda presentan una alta energía efectiva biológica, es decir, son absorbidos por numerosos componentes celulares. La disminución del ozono estratosférico ha causado un incremento del flujo de radiación UV-B sobre la superficie terrestre (Kerr \& McElroy 1993) y los sistemas acuáticos (Villafañe et al. 2003). La radiación UV-A (315-400 nm) puede ejercer un efecto nocivo en el proceso fotosintético (Cullen et al. 1992), sin embargo, se han observado beneficios de esta banda en la fisiología del alga, induciendo procesos fotobiológicos como la actividad fotoliasa para reparar el daño en el ADN (Buma et al. 1997) o estimulando la actividad nitrato reductasa (Viñegla 2000).

Entre los componentes celulares de organismos fotosintéticos marinos que son dañados por la radiación UV-B se pueden citar proteínas, ADN, ARN y membranas (Buma et al. 1995, 1997, Bischof et al. 2000, Vincent \& Neale 2000, Helbling et al. 2001), además algunos procesos biológicos como la fotosíntesis (Cordi et al. 1997, Figueroa et al. 1997), enzimas como Rubisco (Bischof et al. 2002) o nitrato reductasa (Gómez et al. 1998, Figueroa \& Viñegla 2001), crecimiento (Grobe \& Murphy 1994, Altamirano et al. 2000) o la germinación de esporas (Wiencke et al. 2000, Altamirano et al. 2003). A escalas mayores tales efectos pueden configurar cambios en la estructura de las comunidades de macroalgas en los sistemas acuáticos (Bischof et al. 1998, Wiencke et al. 2000).

La sensitividad frente a la radiación UV-B varía según las especies y según los estadios de vida afectados (Dring et al. 1996). Sin embargo, existen mecanismos de protección y reparación frente a la radiación UV. Contra el daño fotoquímico, la fotorreactivación de ADN (mediada por radiación PAR y UV-A), la reparación de la escisión nucleotídica y la recombinación del ADN (Mitchell \& Karentz 1993, Van de Poll et al. 2001), la acumulación de antioxidantes lipo- e hidrosolubles y la actividad de enzimas antioxidantes (Cockell \& Knowland 1999). Otro mecanismo fisicoquímico contra los efectos dañinos de la radiación UV, es la biosíntesis y acumulación de moléculas pantalla. Entre estas sustancias con papel potencial como pantalla de la radiación UV en organismos acuáticos se encuentran los carotenoides, las ficobiliproteínas, los fenoles, las cumarinas y los aminoácidos tipo micosporina (MAAs).

Esta revisión tiene por objetivo analizar de forma crítica la información disponible en la literatura más relevante sobre los MAAs. Su papel fotoprotector será analizado en detalle y se propondrá un posible papel como reservorio de nitrógeno, puesto que recientemente se ha observado una relación del contenido de MAAs con la disponibilidad de nitrógeno inorgánico.

\section{Descubrimiento de los MAAs}

En 1961 Tsujino \& Saito descubrieron sustancias que absorbían la radiación UV en algas rojas: se les denominó "sustancia 334". Sin embargo, las micosporinas fueron caracterizadas por primera vez en el hongo Ascochyta pisi de ahí su nombre y se las relacionó con la inducción lumínica de la esporulación (Leach 1965). Favre-Bonvin et al. (1976) fueron los primeros en describir la estructura química de una micosporina en el hongo Stereum hirsutum y fue denominada micosporina-310.

En los últimos años, se han aislado de cianobacterias, algas y otros organismos una serie de derivados de la micosporina-310. Se trata de derivados imino-carbonil del cromóforo de ciclohexenona de las micosporinas, y se denominan aminoácidos tipo micosporina (MAAs). Tanto las micosporinas como los MAAs tienen conjugado en el anillo sustituyentes nitrogenados (amino), aminoácidos o sus amino-alcoholes correspondientes: la glicina es el aminoácido más común presente en los MAAs. En cuanto a su localización, los MAAs se pueden acumular intracelularmente (García-Pichel \& Castenholz 1993), o extracelularmente, como ha sido descrito en cianobacterias (Böhm et al. 1995). Estos autores 
describieron en Nostoc commune MAAs unidos a oligosacáridos (se denominaron OMAAs) de la cubierta extracelular donde formaban complejos de alto peso molecular unidos a dicha cubierta mediante interacciones no covalentes (Hill et al. 1994).

En organismos marinos hay descritos 23 MAAs: mycosporine-glicina y asterina-330 (Gleason 1993), shinorine (Tsujino et al. 1980), porphyra-334 y palythine (Takano et al. 1978a), palythinol (Dunlap et al. 1986), palythene (Takano et al. 1978b), mycosporinetaurina y mycosporine-2-glicina (Stochaj et al. 1994), ácido palythenico, mycosporineglicina-valina (Karentz et al. 1991b), mycosporine-glicina-treonina, mycosporineglicina-serina, mycosporine-glicina-Asp (Grant et al. 1985), mycosporine-metilaminatreonina (Wu Won et al. 1995), palythineserina, palythine-treonina-sulfato y palythineserina-sulfato (Wu Won et al. 1997), mycosporine-NMA:serina, mycosporineNMA:treonina; mycosporine-Glu-glicina, mycosporine-Glu y usujirene (Bandaranayake et al. 1996) (Fig. 1).

\section{Biosintesis de los MAAs}

La biosíntesis de las micosporinas fue estudiada por primera vez en el hongo Trichothecium roseum (Favre-Bonvin et al. 1987). Mediante la incorporación de moléculas marcadas con ${ }^{14} \mathrm{C}$ se concluyó que el anillo de ciclohexenona se origina en la primera parte de la ruta del ácido shikimico, siendo el 6deoxygadusol el precursor de estos anillos (más evidencias en Bandaranayake \& Rocher 1999, Shick et al. 1999, Portwich \& García-Pichel 2003). Las micosporinas se sintetizan, por lo tanto, en los plástidos y en el citosol. Las cadenas de los MAAs proceden entonces de los correspondientes aminoácidos que se incorporan al anillo en un paso posterior de condensación (Fig. 2).

La ruta del ácido shikimico está presente en bacterias, hongos y plantas (Bentley 1990, Haslam 1993), mientras que la presencia de MAAs en algunos animales hay que atribuirla a la dieta (por consumo de micro o macroalgas que contienen MAAs) (evidencias experimentales en Carroll \& Shick 1996, Carefoot et al. 2000, Newman et al. 2000, Shick et al. 2002). Una vez incorporado los
MAAs al organismo pueden sufrir una serie de reacciones de bioconversión (Dunlap \& Shick 1998).

La ruta del ácido shikimico es vital para el metabolismo intermediario y está unida funcionalmente a la fotosíntesis (Bentley 1990). Por ello se pensó que incrementos en las tasas fotosintéticas podrían incrementar el flujo de hidratos de carbono a través de esta ruta y consecuentemente incrementar la producción de MAAs (Carreto et al. 1990, Glynn et al. 1993, Lesser et al. 1994). Sin embargo, la dependencia no es completa, ya que una alta tasa de biosíntesis de MAAs no siempre estaría asociada a altas tasas de fotosíntesis (Neale et al. 1998). Por ejemplo, en el alga roja Chondrus crispus, la síntesis de MAAs puede aún ocurrir en ausencia de radiación PAR (Karsten et al. 1998a). Por lo tanto, se deduce que la regulación de MAAs no necesariamente está acoplada a la asimilación de carbono fotosintético, sino que la regulación de la síntesis de MAAs es parte de una serie de respuestas a factores ambientales, por ejemplo altas irradiancias (Moisan \& Mitchell 2001), o de procesos fotomorfogénicos (Korbee et al. 2004, 2005).

\section{Propiedades físico-químicas de los MAAs}

Los MAAs poseen características de compuestos iónicos (son anfolitos) con una temperatura de desnaturalización alta y una alta solubilidad en agua. Los pesos moleculares rondan los $330 \mathrm{Da}$. Los máximos de absorción se encuentran en el intervalo de 310-360 nm y sus espectros se caracterizan por mostrar solo un máximo de absorción con un ancho de banda de aproximadamente $20 \mathrm{~nm}$ (Karentz 1994, Carroll \& Shick 1996). Los MAAs poseen una alta fotoestabilidad (Conde et al. 2000) y una baja emisión de fluorescencia (Conde et al. 2000, Shick et al. 2000, Inoue et al. 2002, Misonou et al. 2003); estas últimas propiedades apoyan el papel fotoprotector que les atribuyen algunos autores a los MAAs.

\section{Funciones de los MAAs}

(1) Osmorregulación: en comunidades de cianobacterias halofílicas se ha observado que los MAAs actúan como osmolitos orgánicos y solutos compatibles (Oren 1997). En 
<smiles>CNC1=C(OC)C(=NC2CC(O)(CO)CC(NCC(=O)O)=C2OC)CC(O)(CO)C1</smiles><smiles>C/C=C\N=C1CC(O)(CO)CC(NCC(=O)O)=C1OC</smiles>

Palythene $\mathrm{CO}_{2} \mathrm{H}$ Usujirene ${ }^{\mathrm{CO}_{2} \mathrm{H}}$ Ácido palythenico $\mathrm{CO}_{2} \mathrm{H}$ Palythine-Thr-Sulfato $\lambda_{\text {máx }}=360 \mathrm{~nm} \quad \lambda_{\text {máx }}=357 \mathrm{~nm} \quad \lambda_{\text {máx }}=337 \mathrm{~nm} \quad \lambda_{\text {máx }}=321 \mathrm{~nm}$<smiles>COC1=C(NCC(=O)O)CC(O)(CO)CC1=NCC(=O)O</smiles>

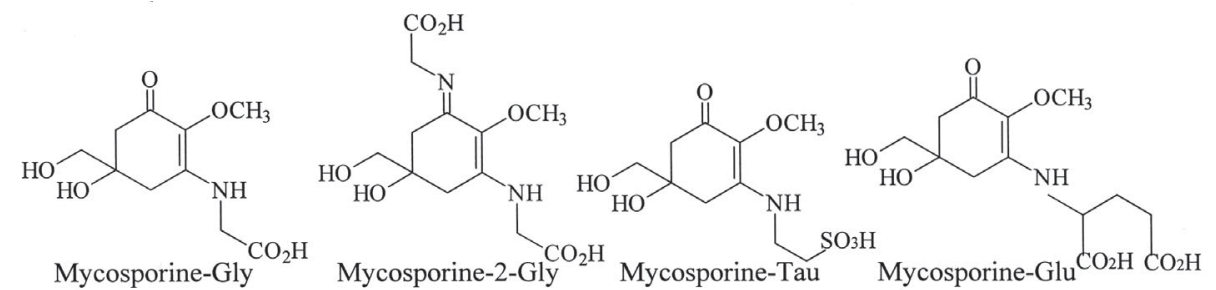
Mycosporine-Gly Mycosporine-2-Gly ${ }^{\mathrm{CO}_{2} \mathrm{H}}$ Mycosporine-Tau Mycosporine-Glu $\mathrm{CO}_{2} \mathrm{H} \mathrm{CO} 2 \mathrm{H}$<smiles>CCN=C1CC(O)(CO)CC(NC(O)C(=O)O)=C1OC</smiles>
$\lambda_{\text {max }}=309 \mathrm{~nm}$<smiles>CNC1=C(OC)C(=NC(C(=O)O)C(C)O)CC(O)(CO)C1</smiles><smiles>COC1=C(NCC(=O)O)CC(O)(CO)CC1=NC(CO)C(=O)O</smiles>

Mycosporine-NMA:Ser Mycosporine-NMA:Thr Mycosporine-metilamina-Thr Mycosporine-Gly-Ser<smiles>COC1=C(NCC(=O)O)CC(O)(CO)CC1=NC(C=[18O])C(C)C</smiles><smiles>COC1=C(NCC(=O)O)CC(O)(CO)CC1N=C(CO)CO</smiles><smiles>COC1=C(NCC(=O)O)CC(O)(CO)CC1=NC(CC(=O)O)CC(=O)O</smiles><smiles>COC1=C(NCC(=O)O)CC(O)(CO)CC1=NC(O[As])C(C)C</smiles>

Mycosporine-Gly-Val Mycosporine-Glu-Gly Mycosporine-Gly-Asp $\lambda_{\text {máx }}=335 \mathrm{~nm}$ $\lambda_{\text {máx }}=330 \mathrm{~nm}$

$\lambda_{\text {max }}=$ ?

Mycosporine-Gly-Thr

Fig.1: Estructuras químicas y máximos de absorción de los 23 MAAs identificados en organismos acuáticos (referencias en el texto).

Chemical structures and absorption maxima of the 23 MAAs identified in aquatic organisms (references in the text) 

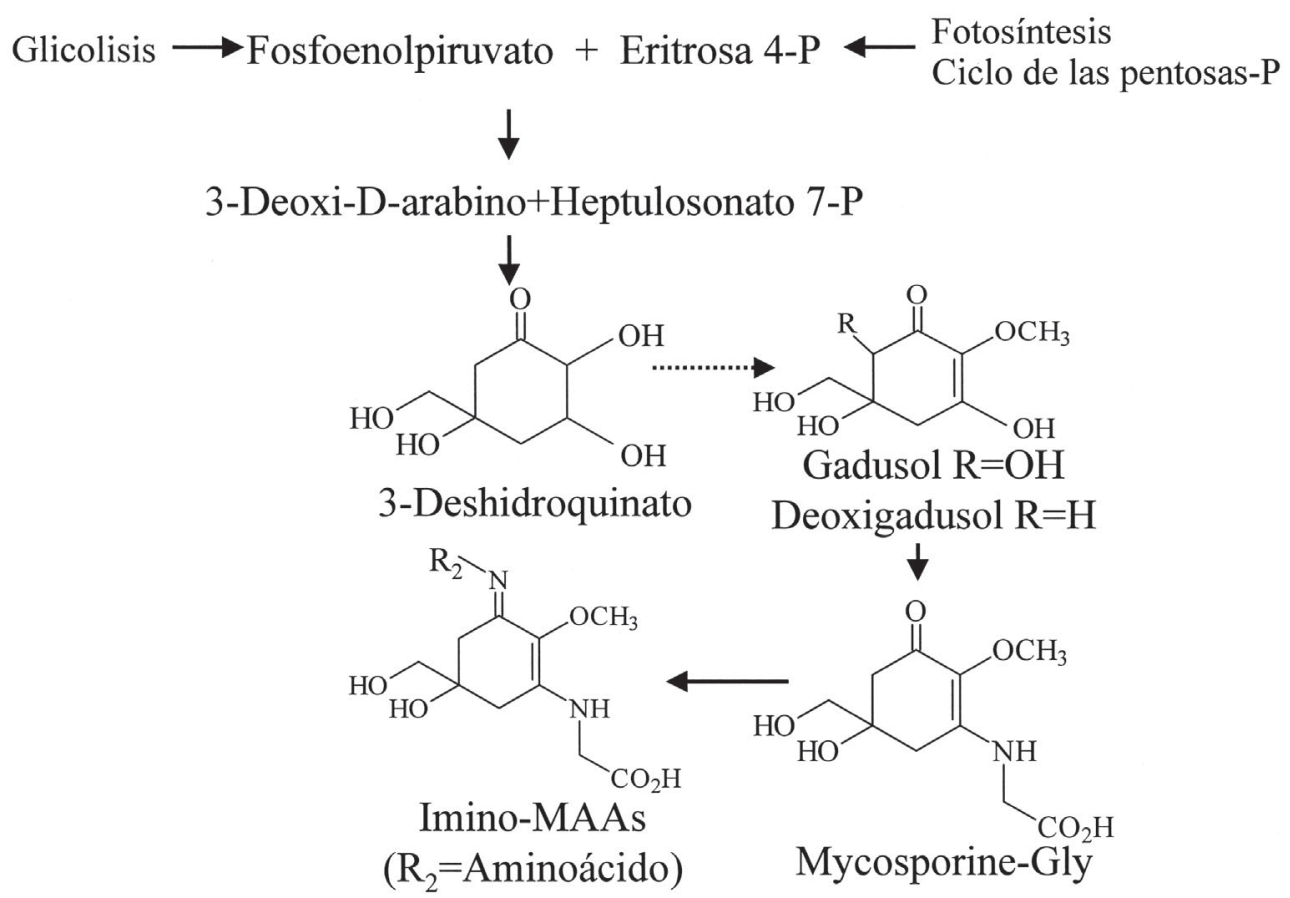

Fig. 2: Posible ruta biosintética de los MAAs a partir de un intermediario de la ruta del ácido shikimico. Modificado de: Portwich \& García-Pichel (2003), Carreto et al. (2005).

Possible MAAs biosynthetic pathway from an intermediate of the shikimic acid pathway. Modified from: Portwich \& García-Pichel (2003), Carreto et al. (2005).

Chlorogloeopsis sp. la síntesis de mycosporineglicina se produce por acción osmótica y además, existe sinergismo con la radiación UVB (Portwich \& García-Pichel 1999). Sin embargo, este resultado fue discutido en un estudio posterior (Shick \& Dunlap 2002), ya que esta cianobacteria tolera concentraciones salinas próximas al $70 \%$ del agua de mar normal, y los MAAs representaban menos del $5 \%$ del total de osmolitos, por lo tanto, bajo esas condiciones el papel fisiológico de los MAAs no parecía ser el de regulación osmótica.

(2) Posible papel en procesos reproductivos: debido a que en los huevos de algunos invertebrados marinos se ha determinado altas concentraciones de MAAs alrededor del periodo de desove se ha sugerido un posible rol en algunos eventos reproductivos (Grant et al. 1985, Post \& Larkum 1993, Adams \& Shick 1996, Bandaranayake et al. 1996, 1997, Bandaranayake \& Rocher 1999). De este modo, la concentración de MAAs y su sincronización podría emplearse como un indicador de la maduración de los ovarios (Shick \& Dunlap 2002). Quedaría por demostrar si los factores medioambientales y fisiológicos que regulan el desarrollo de los ovarios también controlan el secuestro y el metabolismo de los MAAs.

(3) Antioxidación: se demostró que mycosporine-glicina poseía actividad antioxidante in vitro (Dunlap \& Yamamoto 1995). Recientemente se ha determinado la capacidad que posee esta molécula de proteger sistemas biológicos capturando los radicales de oxígeno generados por estrés oxidativo en Escherichia coli (Suh et al. 2003). Esta propiedad podría estar relacionada con la capacidad de los MAAs de bloquear la producción de fotodímeros de timina, como el 6-4 y 5-6, como ha sido reportado para Porphyra yezoensis (Misonou et al. 2003). Se ha propuesto un amortiguamiento del estado de excitación de las moléculas de timina mediante un proceso de transferencia energética directa a los MAAs, relajándose posteriormente estas y disipando calor (Misonou et al. 2003). En este sentido, se ha publicado una patente en la que 
se propone emplear los MAAs en cosméticos contra el envejecimiento prematuro de la piel, gracias a su capacidad antioxidante (Schmid et al. 2004).

(4) Pigmento accesorio: la idea que los MAAs actúan como pigmentos ha sido recurrente en la literatura y van desde una función como precursores de algunos pigmentos (Price \& Forrest 1969, Shibata 1969) hasta un rol fotosintético: debido a que la emisión de fluorescencia de los MAAs a 430$450 \mathrm{~nm}$ podría permitir a la clorofila del sistema fotosintético aprovechar la energía de la luz absorbida en la región del UV, se ha pensado que los MAAs estarían actuando como pigmentos accesorios (Price \& Forrest 1969, Maragos 1972, Sivalingam et al. 1976a, 1976b, Logan et al. 1990). Sin embargo, en un estudio reciente en la microalga Phaeocystis antarctica se ha demostrado que los MAAs no transfieren la energía de la radiación UV (directamente o por fluorescencia) a la clorofila $a$, y por lo tanto, no participarían en la fotosíntesis (Moisan \& Mitchell 2001). Es más, del estudio in vitro de las propiedades fotofísicas de algunos MAAs, como shinorine y porphyra-334 (Conde et al. 2000, 2004, Shick et al. 2000) se desprende que su rendimiento de transferencia de energía a otras moléculas es muy bajo. La disipación térmica sería el mecanismo por el que liberan la energía de la radiación UV absorbida, sin producir residuos de la fotolisis de las moléculas.

\section{Función fotoprotectora}

En general, gran parte de la evidencia experimental apoya la idea que una de las principales funciones de los MAAs es de fotoprotección, especialmente por su capacidad para absorber a longitudes de onda corta. Esto unido a su alta fotoestabilidad in vitro (Conde et al. 2000, 2004) e in vivo (Adams \& Shick 2001) los convierten en efectivos agentes protectores frente a la radiación UV. Diversos tipos de organismos acuáticos han sido utilizados para estudiar las propiedades y funciones de los MAAs.

(1) Microalgas y cianobacterias: en microalgas eucarióticas y cianobacterias la acumulación de MAAs en presencia de radiación UV (solar o artificial) es especiedependiente y depende del tipo de MAA, estaría controlada por la calidad espectral de la radiación incidente (Carreto et al. 1990, GarcíaPichel et al. 1993, Lesser 1996, Helbling et al. 1996, Riegger \& Robinson 1997, Wängberg et al. 1997, Hannach \& Sigleo 1998, Klisch \& Häder 2000, Sinha et al. 2003). Se ha atribuido la absorción en el rango de radiación UV en aguas oceánicas a los MAAs presentes en el fitoplancton (Vernet et al. 1989, Helbling et al. 1996). En el dinoflagelado Lingulodinium polyedrum, la radiación UV provoca un descenso de la concentración de MAAs dentro de la célula y un incremento en la absorción de radiación UV en el medio circundante (Vernet \& Whitehead 1996). En los últimos años se ha confirmado este hecho, demostrándose que los MAAs son un componente de la MOD (materia orgánica disuelta) que aun estando presente en bajas concentraciones, contribuyen a la absorción de la radiación UV (Whitehead \& Vernet 2000).

Respecto a la capacidad de fotoprotección de los MAAs, las células de fitoplancton de gran tamaño y esféricas, con bajo autosombreado, se beneficiarían más de la inversión en la síntesis de productos pantalla. A esta conclusión se llegó empleando modelos ópticos y asumiendo que los MAAs se distribuían de manera homogénea en la célula (García-Pichel 1994, 1996). En este sentido, los MAAs serían eficientes protectores de la radiación UV únicamente en los afloramientos o en las grandes colonias de fitoplancton antártico (Bracher \& Wiencke 2000). En el dinoflagelado Gymnodinium sanguineum los MAAs protegen al menos en un $80 \%$ de daños provocados por la radiación UV en la actividad fotosintética (Neale et al. 1998). Sin embargo, el papel fotoprotector de los MAAs no queda demostrado en la forma colonial de la Prymnesiofita antártica Phaeocystis Antarctica, ya que pese a acumular MAAs, su crecimiento se mantiene susceptible a la radiación UV-B (Karentz \& Spero 1995). Por otro lado, algunas especies de diatomeas antárticas no acumulan MAAs y sobreviven mejor a la radiación UV-B que $P$. antarctica (Davidson et al. 1994).

Algunos autores han sugerido que en cianobacterias, los MAAs actúan como un importante mecanismo de protección frente al daño producido por la radiación UV (Karentz et al. 1991a, 1991b, Ehling-Schulz et al. 1997, Quesada et al. 1999). Sin embargo, esta 
protección depende de la localización de estos pigmentos y de la especie en cuestión. Asumiendo una localización citoplasmática de los MAAs, la protección que brindarían a las cianobacterias estaría entre un 10 y un $30 \%$ (García-Pichel \& Castenholz 1993). Sin embargo, la capacidad de fotoprotección sería superior cuando se trata de comunidades o colonias de cianobacterias (Castenholz 1997).

(2) Macroalgas: diversos estudios apuntan hacia el papel fotoprotector de los MAAs en macroalgas. Las especies de zonas templadas expuestas a altas dosis de radiación solar presentan una concentración intracelular de MAAs más alta que otras especies de altas latitudes y algas de aguas profundas. Los MAAs están presentes en algas rojas, mientras que algas verdes y pardas no tienen MAAs o bien solo contienen trazas de ellos (Karentz et al. 1991b, Karentz 1994, Molina \& Montecino 1996, Karsten et al. 1998b, Hoyer et al. 2001, Huovinen et al. 2004, Korbee-Peinado 2004). En general, la concentración de MAAs en macroalgas depende de varios factores: (a) se ha determinado una dependencia de la cantidad de radiación recibida, tanto tras exponer en aguas someras ejemplares de algas rojas de aguas profundas, como tras analizar la dependencia estacional en la acumulación (Post \& Larkum 1993, Karsten et al. 1999, Karsten \& West 2000), (b) en varias especies, existe una acumulación diferencial a lo largo del talo. Por ejemplo, los ápices de las especies árticas Devaleraea ramentacea y Palmaria palmata contienen cinco veces más MAAs que la base (Karsten \& Wiencke 1999, Karsten et al. 1999), (c) se ha sugerido que mayores niveles de MAAs podrían conferir ventajas ecológicas, como ha sido reportado en el alga roja Mastocarpus stellatus, cuyo mayor contenido de MAAs podría ser la causa de la mayor resistencia y competitividad frente a otra alga roja como es Chondrus crispus (Bischof et al. 2000), (d) los contenidos de MAAs podrían ser regulados por factores intrínsecos. En el alga roja Porphyra umbilicalis no se estimulaba la acumulación de MAAs ni con radiación PAR ni con UV (A y B), quizá debido a que los niveles de MAAs se mantenían altos (en saturación) desde el inicio del experimento (Gröniger et al. 1999). Tampoco se ha detectado una inducción de la síntesis de
MAAs en el alga roja Gracilaria cornea (Sinha et al. 2000). Y mientras que en $D$. ramentacea se encontró una correlación positiva entre el incremento de la concentración de MAAs y la resistencia fotosintética frente a la radiación $U V$; en $P$. palmata aun caracterizándose por una concentración de MAAs similar, no se encontró tal relación (Karsten et al. 2003). Aparentemente, es posible distinguir tres tipos de macroalgas en relación con su contenido de MAAs: (i) especies que no pueden sintetizar MAAs, (ii) especies que poseen una concentración basal de MAAs que puede variar según la radiación recibida, y (iii) especies con una concentración interna constitutivamente alta que se mantiene constante independientemente de las condiciones ambientales (Hoyer et al. 2001).

(3) Animales marinos: especies de zooplancton de la Patagonia argentina (Daphnia menucoensis y Metacyclops mendocinus) acumulan MAAs que parecen contribuir en su adaptación a las elevadas dosis de radiación UV recibidas en su hábitat natural (Gonçalves et al. 2002). Se han identificado MAAs en corales (Dunlap \& Chalker 1986), los cuales podrían actuar como moléculas fotoprotectoras ya que el contenido interno disminuye logarítmicamente conforme se incrementa la profundidad (Dunlap et al. 1986). Y aunque en otros trabajos no se ha encontrado tal variación (Shick et al. 1991), los corales poseen una gran plasticidad en su capacidad de absorber la radiación UV (Gleason \& Wellington 1993, Muszynski et al. 1998), según las especies (Teai et al. 1997) o incluso entre dos morfologías de distinto color dentro de una misma especie (Gleason 1993). Actualmente existen diversos trabajos en los que se ha observado que la producción y acumulación de MAAs en corales se induce en presencia de radiación UV (Jokiel \& York 1982, Dunlap \& Shick 1998, Lesser 2000). En ovarios y huevos del erizo marino Strongylocentrotus droebachiensis se ha demostrado la protección que brindan los MAAs frente al estrés oxidativo provocado por la radiación UV (Adams \& Shick 1996). Y en Holothuria atra se observó que algunos MAAs poseían papel fotoprotector (Bandaranayake \& Rocher 1999). Sin embargo otros factores tales como el movimiento del 
agua, pueden modificar la capacidad de acumulación de MAAs en corales (Jokiel et al. 1997, Banaszak et al. 1998).

Además del papel fotoprotector de los MAAs en los organismos que los acumulan, la acumulación de MAAs puede proteger a algas endosimbióticas de algunos animales marinos (Dunlap \& Shick 1998). De hecho, la presencia de MAAs en la superficie de la túnica de una ascidia colonial protege a sus simbiontes fotoautótrofos del daño producido por la radiación UV (Dionisio-Sese et al. 1997). Concretamente en la almeja gigante Tridacna crocea, los MAAs absorben el $87 \%$ de la radiación de $310 \mathrm{~nm}$ y un $90 \%$ la de $320 \mathrm{~nm}$ antes de que esta radiación alcanza una profundidad de $0,2 \mathrm{~mm}$ en el manto sifonal (Ishikura et al. 1997). Sin embargo, existen resultados que no apoyan el papel de los MAAs como barrera frente al daño en sus algas simbiontes; en el coral Hawaiano Montipora verrucosa, a pesar de que se producía un incremento en la concentración de MAAs, la fotosíntesis de sus algas simbiontes decrecía tras la exposición a radiación UV (Kinzie 1993).

Tomando en cuenta que los MAAs se encuentran ampliamente distribuidos, es posible argumentar que estos compuestos han tenido un rol fundamental en la evolución de las características fotobiológicas de los organismos marinos. Ya que la vida se originó bajo condiciones de ausencia de ozono y, por tanto, en escenarios de alta radiación UV-B, la protección frente a la exposición a la radiación UV ha debido ser un importante carácter en la evolución de los primeros eucariotas y, por lo tanto, en el proceso de selección natural. Aunque no hay evidencias del origen filogenético de los MAAs ni de los patrones de distribución en los distintos grupos taxonómicos, son posibles diversas afinidades entre grupos de organismos: por ejemplo, las rutas de síntesis de MAAs son muy similares en hongos y cianobacterias. Los pigmentos fósiles presentes en los sedimentos de lagos boreales pueden emplearse para documentar cambios de radiación UV a lo largo de la historia, estos pigmentos son principalmente escitoneminas, compuestos producidos por las algas bentónicas con espectros de absorción muy similares a los de los MAAs (Leavitt et al. 1997).

\section{Otras posibles funciones}

Recientemente se ha determinado que no solo la calidad y cantidad de energía recibida influyen en la síntesis y acumulación de MAAs, sino que también influye la disponibilidad de nitrógeno en el medio de cultivo (Litchman et al. 2002, Korbee et al. 2004, 2005). En dinoflagelados se ha observado que la concentración de MAAs es inferior cuando en el medio de cultivo hay menos nitrato, lo cual estaría asociado a un incremento de la sensibilidad de la fotosíntesis ante la inhibición por la radiación UV. Debido a una menor eficiencia de reparación, como principal mecanismo, aunque también podría estar asociado a un menor tamaño celular y a una menor concentración de MAAs (Litchman et al. 2002). En Porphyra columbina a mayor concentración de amonio en el medio de cultivo, mayor acumulación de MAAs, lo cual está en función de la radiación incidente, siendo más efectiva la radiación UV-A (Korbee et al. 2004). La acumulación de MAAs en alta disponibilidad de nitrógeno se ha observado en otras especies como Porphyra umbilicalis, $P$. leucosticta y Grateloupia lanceola (Tabla 1). Los MAAs podrían por lo tanto actuar no solo como sustancias fotoprotectoras con cierta capacidad antioxidante, sino también ser reservorio de nitrógeno pudiendo removilizarse en periodos de baja concentración de nutrientes. Este posible rol como reservorio de nitrógeno podría ser análogo al rol sugerido para otros pigmentos tales como las biliproteínas que pueden actuar como un pigmento accesorio pero además como fuente de proteínas de reserva (Algarra \& Rüdiger 1993, Tandeau de Marsac \& Houmard 1993, Talarico \& Maranzana 2000).

En los últimos años se ha evaluado la capacidad fotoprotectora de los MAAs en organismos acuáticos, tanto de aguas polares como templadas y tropicales y se ha avanzado en el conocimiento sobre la biosíntesis y fotorregulación de estos compuestos, pero los trabajos recientes sobre el papel del nitrógeno inorgánico amplían aún más la funcionalidad de estas sustancias en la aclimatación frente a los cambios ambientales. La capacidad fotoprotectora se une a otras como la capacidad antioxidante, osmorregulación, posible reservorio de nitrógeno, etc. Es decir, los MAAs 


\section{TABLA 1}

Concentraciones iniciales de aminoácidos tipo micosporina (MAAs) e incrementos temporales, expresados en $\mathrm{mg} \mathrm{g}^{-1} \mathrm{PS}$, en las algas rojas Porphyra umbilicalis, P. leucosticta, P. columbina y Grateloupia lanceola, bajo diferentes concentraciones de amonio $(0,50 / 100$ y $300 \mu \mathrm{M})$. Fuentes: ${ }^{1}$ Korbee et al. (2005), ${ }^{2}$ Korbee et al. (2004), ${ }^{3}$ Huovinen, Matos, Sousa-Pinto \& Figueroa (resultados no publicados)

Initial concentrations of mycosporine-like amino acids (MAAs) and temporal increments, expressed as $\mathrm{mg} \mathrm{g}^{-1} \mathrm{DW}$ in the red algae Porphyra umbilicalis, P. leucosticta, P. columbina and Grateloupia lanceola, under different ammonium concentrations $(0,50 / 100$ and $300 \mu \mathrm{M})$. Sources: ${ }^{1}$ Korbee et al. (2005), ${ }^{2}$ Korbee et al. (2004), ${ }^{3}$ Huovinen, Matos, SousaPinto \& Figueroa (unpublished results)

\begin{tabular}{|c|c|c|c|c|c|}
\hline Días de cultivo & $\begin{array}{l}\text { Concentración de } \\
\text { amonio }(\mu \mathrm{M})\end{array}$ & P. umbilicalis ${ }^{l}$ & P. leucosticta ${ }^{l}$ & P. columbina ${ }^{2}$ & $\begin{array}{c}\text { Grateloupia } \\
\text { lanceola }^{3}\end{array}$ \\
\hline Inicial & & $9,23 \pm 1,40$ & $6,99 \pm 1,62$ & $5,49 \pm 0,99$ & $3,25 \pm 0,75$ \\
\hline Tres & $\begin{array}{c}50 / 100 \\
300\end{array}$ & $\begin{array}{l}0,12 \\
0,36\end{array}$ & $\begin{array}{l}0,27 \\
0,51\end{array}$ & $\begin{array}{l}0,78 \\
2,28\end{array}$ & $\begin{array}{l}0,8 \\
1,3\end{array}$ \\
\hline Seis/siete & $\begin{array}{c}50 / 100 \\
300\end{array}$ & $\begin{array}{c}1,85 \\
2,9\end{array}$ & $\begin{array}{l}0,91 \\
5,39\end{array}$ & $\begin{array}{l}1,24 \\
5,14\end{array}$ & \\
\hline
\end{tabular}

parecen sustancias multifuncionales como otros metabolitos secundarios, por ejemplo, las sustancias fenólicas de algas pardas.

\section{CONCLUSIONES}

Desde que se detectó la presencia del agujero de ozono antártico se ha invertido mucho esfuerzo en determinar cuáles son los efectos que provoca el incremento de la radiación UV sobre la superficie terrestre y ecosistemas acuáticos. Los antecedentes citados en esta revisión indican que los MAAs se encuentran en multitud de organismos marinos donde cumplen un rol fotoprotector ante la radiación UV. La gran cantidad de datos existentes apuntan a una relación entre el contenido interno de MAAs y la cantidad de radiación UV recibida ya sea por distinta localización batimétrica o latitudinal. Aunque no existen muchas evidencias experimentales que lo demuestren, en general se ha concluido que los MAAs protegen solo parcialmente a los componentes celulares y procesos fisiológicos (Wood 1989, García-Pichel et al. 1993, Post \& Larkum 1993, Lesser 1996, Neale et al. 1998, Franklin et al. 1999, Sinha et al. 2000, Yakovleva \& Titlyanov 2001).

Las perspectivas de estudios futuros en el campo de los MAAs son promisorias. Para evaluar con más profundidad la función fotoprotectora de los MAAs se debe realizar un estudio pormenorizado de cuestiones que quedan aún por resolver, como la localización, la regulación de la biosíntesis, los mecanismos de regulación a nivel de transcripción o transducción que intervienen como respuesta a la radiación UV y/o disponibilidad de nitrógeno inorgánico. La respuesta a estas preguntas ayudaría a una mejor interpretación de los resultados obtenidos en los estudios de la acumulación de MAAs en función a la calidad y cantidad de radiación y a la disponibilidad de amonio.

\section{AGRADECIMIENTOS}

Este trabajo ha sido financiado por el Ministerio de Educación y Ciencia de España (Proyecto DEPUSIAL AGL 2001-1888-C03-02 y beca de investigación de N.K.P.).

\section{LITERATURA CITADA}

ADAMS NL \& JM SHICK (1996) Mycosporine-like amino acids provide protection against ultraviolet radiation in eggs of the green sea urchin Strongylocentrotus droebachiensis. Photochemistry and Photobiology 64: 149-158.

ADAMS NL \& JM SHICK (2001) Mycosporine-like amino acids prevent UVB-induced abnormalitites during early development of the green see urchin Strongylocentrotus droebachiensis. Marine Biology 138: 267-280. 
ALGARRA P \& W RÜDIGER (1993) Acclimation processes in the light harvesting complex red alga Pophyridium purpureum (Bory) Drew et Ross according to irradiance and nutrient availability. Plant Cell \& Environment 16: 149-159.

ALTAMIRANO M, A FLORES-MOYA \& FL FIGUEROA (2000) Long-term effect of natural sunlight under various ultraviolet conditions on growth and photosynthesis of intertidal Ulva rigida (Chlorophyceae) culturated in situ. Botanica Marina 43: 119-126.

ALTAMIRANO M, A FLORES-MOYA \& FL FIGUEROA (2003) Effects of UV radiation and temperature on growth germlings of three species of Fucus (Phaeophyceae). Aquatic Botany 75: 9-20.

BANASZAK AT, MP LESSER, IB KUFFNER \& M ONDRUSEK (1998) Relationship between ultraviolet light (UV) radiation and Mycosporinelike amino acids (MAAs) in marine organisms. Bulletin of Marine Science 63: 617-628.

BANDARANAYAKE WM \& A DES ROCHER (1999) Role of secondary metabolites and pigments in epidermal tissues, ripe ovaries, viscera, gut contents and diet of the sea cucumber Holothuria atra. Marine Biology 133: 163-169.

BANDARANAYAKE WM, JE BEMIS \& DJ BOURNE (1996) Ultraviolet absorbing pigments from the marine sponge Dysidea herbacea: isolation and structure of a new mycosporine. Comparative Biochemistry Physiology C 115: 281-286.

BANDARANAYAKE WM, DJ BOURNE \& RG SIM (1997) Chemical composition during maturing and spawning of the sponge Dysidea herbacea (Porifera: Demospongiae). Comparative Biochemistry Physiology C 118: 851-859.

BENTLEY R (1990) The shikimate pathway- a metabolic tree with many branches. Critical Reviews in Biochemistry and Molecular Biology 25: 307-384.

BISCHOF K, D HANELT \& C WIENCKE (1998) UVradiation can affect depth-zonation of Antarctic macroalgae. Marine Biology 131: 597-605.

BISCHOF K, G KRÄBS, D HANELT \& C WIENCKE (2000) Photosynthetic characteristics and mycosporine-like amino acids under UV radiation: a competitive advantage of Mastocarpus stellatus over Chondrus crispus at the Helgoland shoreline? Helgoland Marine Research 54: 47-52.

BISCHOF K, G KRABS, C WIENCKE \& D HANELT (2002) Solar ultraviolet radiation affects the activity of ribulose-1, 5-bisphosphate carboxylaseoxygenase and the composition of photosynthetic and xanthophyll cycle pigments in the intertidal green alga Ulva lactuca. Planta 215: 502-509.

BÖHM GA, W PFLEIDERER, P BÖGER \& S SCHERER (1995) Structure of a novel oligosaccharidemycosporine-amino acid ultraviolet A/B sunscreen pigment from the terrestial cyanobacterium Nostoc commune. Journal of Biological Chemistry 270: 8536-8539.

BRACHER AU \& C WIENCKE (2000) Simulation of the effects of naturally enhanced UV radiation on photosynthesis of Antarctic phytoplankton. Marine Ecology Progress Series 196: 127-141.

BUMA AGJ, EJ VAN HANNEN, L VAN, MJ ROZA, W VELDHUIS \& WWC GIESKES (1995) Monitoring ultraviolet B-induced DNA damage in individual diatom cells by immunofluorescent thymine dimer detection. Journal of Phycology 31: 314-321.

BUMA AGJ, AH ENGELEN \& WWC GIESKES (1997) Wavelength-dependent induction of thymine dimers and growth rate reduction in the marine diatom Cyclotella sp. exposed to ultraviolet radiation. Marine Ecology Progress Series 153: 91-97.

CAREFOOT TH, D KARENTZ, SC PENNINGS \& CL YOUNG (2000) Distribution of mycosporine-like amino acids in the sea hare Aplysia dactylomela: effect of diet on amounts and types sequestered over time in tissues and spawn. Comparative Biochemistry and Physiology C 126: 91-104.

CARRETO JI, MO CARIGNAN, D DALEO, \& SG DE MARCO (1990) Occurrence of mycosporine-like amino acids in the red-tide dinoflagellate Alexandrium excavantum: UV-protoprotective compounds? Journal of Plankton Research 12: 909 922.

CARRETO JI, MO CARIGNAN \& NG MONTOYA (2005) A high-resolution reverse-phase liquid chromatography method for the analysis of mycosporine-like amino acids (MAAs) in marine organisms. Marine Biology 146: 237-252.

CARROLL AK \& JM SHICK (1996) Dietary accumulation of UV-absorbing mycosporine-like amino acids (MAAs) by the green sea urchin (Strongylocentrotus droebachiensis). Marine Biology 124: 561-569

CASTENHOLZ RW (1997) Multiple strategies for UV tolerance in cyanobacteria. The Spectrum 10: 10-16.

COCKELL CS \& J KNOWLAND (1999) Ultraviolet radiation screening compounds. Biological Reviews 74: 311-345.

CONDE FR, MS CHURIO \& CM PREVITALI (2000) The photoprotector mechanism of mycosporine-like amino acids. Excited-state properties and photostability of porphyra-334 in aqueous solution. Journal of Photochemistry and Photobiology 56: 139-144.

CONDE FR, MS CHURIO \& CM PREVITALI (2004) The deactivation pathways of the excited-states of the mycosporine-like amino acids shinorine and porphyra-334 in aqueous solution. Photochemical and Photobiological Sciences 3: 960-967.

CORDI B, MH DEPLEDGE, DN PRICE, LF SALTER, \& ME DONKIN (1997) Evaluation of chlorophyll fluorescence, in vivo spectrophotometric pigment absorption and ion leakage as biomarkers of UV-B exposure in marine macroalgae. Marine Biology 130: $41-49$.

CULLEN JJ, PJ NEALE \& MP LESSER (1992) Biological weighting function for the inhibition of phytoplankton photosynthesis by ultraviolet radiation. Science 258: 646-650.

DAVIDSON AT, D BRAMICH, HJ MARCHANT \& A MCMINN (1994) Effects of UVB irradiation on growth and survival of Antarctic marine diatoms. Marine Biology 119: 507-515.

DIONISIO-SESE ML, M ISHIKURA, T MARUYAMA \& S MIYACHI (1997) UV absorbing substances in the tunic of a colonial ascidian protect its symbiont, Prochloron sp., from damage by UV-B radiation. Marine Biology 128: 455-461.

DRING MJ, V MAKAROV, E SCHOSCHINA, M LORENZ \& K LÜNING (1996) Influence of ultraviolet-radiation on chlorophyll fluorescence and growth in different life-history stages of three species of Laminaria (Phaeophyta). Marine Biology 126: $183-191$

DUNLAP WC \& BE CHALKER (1986) Identification and quantification of near-UV absorbing compounds ( $S$ 320) in a hermatypic scleractinian. Coral Reefs 5: 155-159. 
DUNLAP WC \& Y YAMAMOTO (1995) Small-molecule antioxidants in marine organisms-antioxidant activity of mycosporine-glycine. Comparative Biochemistry Physiology B 112: 105-114.

DUNLAP WC \& JM SHICK (1998) UV radiation absorbing mycosporine-like amino acids in coral reef organisms: a biochemical and environmental perspective. Journal of Phycology 34: 418-430.

DUNLAP WC, BE CHALKER \& JK OLIVER (1986) Bathymetric adaptations of reef-building corals at Davies Reef. Australia. III. UV-B absorbing compounds. Journal of Experimental Marine Biology and Ecology 104: 239-248.

EHLING-SCHULZ M, W BILGER \& S SCHERER (1997) UV-B-induced synthesis of photoprotective pigments and extracellular polysaccharides in the terrestrial cyanobacterium Nostoc commune. Journal of Bacteriology 179: 1940-1945.

FAVRE-BONVIN J, N ARPIN \& C BREVARD (1976) Structure de la mycosporine (P-310). Canadian Journal of Chemical 54: 1105-1113.

FAVRE-BONVIN J, J BERNILLON, N SALIN \& N ARPIN (1987) Biosynthesis of mycosporine: mycosporine-glutaminol in Trichothecium roseum. Phytochemistry 26: 2509-2514.

FIGUEROA, FL \& B VIÑEGLA (2001) effects of solar UV radiation on photosynthesis and enzyme activities (carbonic anhydrase and nitrate reductase) in marine macroalgae from Southern Spain. Revista Chilena de Historia Natural 74: 237-249.

FIGUEROA FL, S SALLES, J AGUILERA, C JIMÉNEZ, J MERCADO, B VIÑEGLA, A FLORES-MOYA \& M ALTAMIRANO (1997) Effects of solar radiation on photoinhibition and pigmentation in the red alga Porphyra leucosticta. Marine Ecology Progress Series 151: 81-90.

FRANKLIN LA, I YAKOVLEVA, U KARSTEN \& K LÜNING (1999) Synthesis of mycosporine-like amino acids in Chondrus crispus (Florideophyceae) and the consequences for sensitivity to ultraviolet B radiation. Journal of Phycology 35: 682-693.

GARCÍA-PICHEL F (1994) A model for internal selfshading in planktonic organism and its implications for the usefulness of ultraviolet sunscreens. Limnology and Oceanography 39: 1704-1717

GARCÍA-PICHEL F (1996) The absorption of ultraviolet radiation by microalgae: simple optics and photobiological implications. Scientia Marina 60: 73-79.

GARCÍA-PICHEL F \& RW CASTENHOLZ (1993) Occurrence of UV-absorbing mycosporine-like compounds among cyanobacterial isolates and an estimate of their screening capacity. Applied and Environmental Microbiology 59: 163-169.

GARCÍA-PICHEL F, CE WINGARD \& RW CASTENHOLZ (1993) Evidence regarding the UV sunscreen role of a mycosporine-like compound in the Cyanobacterium Gloeocapsa sp. Applied and Environmental Microbiology 59: 170-176.

GLEASON DF (1993) Differential effects of ultraviolet radiation on green and brown morphs of the Caribbean coral Porites astreoides. Limnology and Oceanography 38: 1452-1463.

GLEASON DF \& GM WELLINGTON (1993) Ultraviolet radiation and coral bleaching. Nature 365: 836838.

GLYNN PW, R IMAI, K SAKAI, Y NAKANO \& K YAMAZATO (1993) Experimental responses of Okinawan (Ryukyu Islands, Japan) reef corals to high sea temperature and UV radiation. Proceedings of the 7th International Coral Reef Symposium University of Guam Press, Guam, USA 1: 27-37.

GÓMEZ I, E PÉREZ-RODRÍGUEZ, B VIÑEGLA, FL FIGUEROA \& U KARSTEN (1998) Effects of solar radiation on photosynthesis, UV-absorbing compounds and enzyme activities of the green alga Dasycladus vermicularis from southern Spain. Journal of Photochemistry and Photobiology B: Biology 47: 46-57.

GONÇALVES RJ, VE VILLAFAÑE \& EW HELBLING (2002) Photorepair activity and protective compounds in two freshwater zooplankton species (Daphnia menucoensis and Metacyclops mendocinus) from Patagonia, Argentina. Photochemical and Photobiological Sciences 1: 996-1000

GRANT PT, C MIDDLETON, PA PLANCK \& RH THOMSON (1985) The isolation of four aminocyclohexenimines (mycosporines) and a structurally related derivative of cyclohexane-1,3dione (gadusol) from the brine shrimp Artemia. Comparative Biochemistry and Physiology B 80: 755-759.

GROBE CW \& TM MURPHY (1994) Inhibition of growth of Ulva expansa (Chlorophyta) by ultraviolet-B radiation. Journal of Phycology 30: 783-790.

GRÖNIGER A, C HALLIER \& DH HÄDER (1999) Influence of UV radiation and visible light on Porphyra umbilicalis: Photoinhibition and MAA concentration. Journal of Applied Phycology 11: 437-445.

HANNACH G \& AC SIGLEO (1998) Photoinduction of UV- absorbing compounds in six species of marine phytoplankton. Marine Ecology Progress Series 174: 207-222.

HASLAM E (1993) Shikimic acid: metabolism and metabolites. Wiley \& Sons, New York, New York, USA. 387 pp.

HELBLING EW, BE CHALKER, WC DUNLAP, O HOLM-HANSEN \& VE VILLAFAÑE (1996) Photoacclimation of Antarctic marine diatoms to solar ultraviolet radiation. Journal of Experimental Marine Biology and Ecology 204: 85-101.

HELBLING EW, VE VILLAFAÑE, AGJ BUMA, M ANDRADE \& F ZARATTI (2001) DNA damage and photosynthetic inhibition induced by solar ultraviolet radiation in tropical phytoplankton (Lake Titicaca, Bolivia). European Journal of Phycology 36: 157-166.

HILL DR, SL HLADUN, S SCHERER \& M POTTS (1994) Water stress proteins of Nostoc commune (Cyanobacteria) are secreted with UV-A/Babsorbing pigments and associate with 1,4-b-Dxylanxylanohydrolase activity. Journal of Biological Chemistry 269: 7726-7734.

HOYER K, U KARSTEN, T SAWALL \& C WIENCKE (2001) Photoprotective substances in Antarctic macroalgae and their variation with respect to depth distribution, different tissues and developmental stages. Marine Ecology Progress Series 211: 117-129.

HUOVINEN P, I GÓMEZ, FL FIGUEROA, N ULLOA, V MORALES \& C LOVENGREEN (2004) Ultravioletabsorbing mycosporine-like amino acids in red macroalgae from Chile. Botanica Marina 47: 21-29.

INOUE Y, H HORI, T SAKURAI, Y TOKITOMO, J SAITO \& T MISONOU (2002) Measurement of fluorescence quantum yield of ultraviolet-absorbing substance extracted from red alga: Porphyra yezoensis and its photothermal spectroscopy. Optical Review 9: 75-80. 
ISHIKURA M, C KATO \& T MARUYAMA (1997) UVabsorbing substances in zooxanthellate and azooxanthellate clams. Marine Biology 128: 649.

JOKIEL PL \& RH YORK (1982) Solar ultraviolet photobiology of the reef coral Pocillopora damicornis and symbiotic zooxanthellae. Bulletin of Marine Science 32: 301-315.

JOKIEL PL, MP LESSER \& ME ONDRUSEK (1997) UVabsorbing compounds in the coral Pocillopora damicornis: interactive effects of UV radiation, photosynthetically active radiation and water flow. Limnology and Oceanography 42: 1468-1473.

KARENTZ D (1994) Ultraviolet tolerance mechanisms in Antarctic marine organisms. Antarctic Research Series 62: 93-110.

KARENTZ D \& HJ SPERO (1995) Response of natural Phaeocystis population to ambient fluctuations of UVB radiation caused by Antarctic ozone depletion. Journal of Plankton Research 17: 1771-1789.

KARENTZ D, J CLEAVER \& DL MITCHELL (1991a) Cell survival characteristics and molecular responses of Antarctic phytoplankton to ultravioletB radiation. Journal of Phycology 27: 326-341.

KARENTZ D, FS MCEUEN, MC LAND \& WC DUNLAP (1991b) Survey of mycosporine-like aminoacids in Antarctic marine organisms: potential protection from ultraviolet exposure. Marine Biology 108: 157-166.

KARSTEN U \& C WIENCKE (1999) Factors controlling the formation of UV-absorbing mycosporine-like amino acids in the marine red alga Palmaria palmata from Spitsbergen (Norway). Journal of Plant Physiology 155: 407-415.

KARSTEN U \& JA WEST (2000) Living in the intertidal zone-seasonal effects on heterosides and sun-screen compounds in the red alga Bangia atropurpurea (Bangiales). Journal of Experimental Marine Biology and Ecology 254: 221-234.

KARSTEN U, LA FRANKLIN, K LÜNING \& C WIENCKE (1998a) Natural ultraviolet and photosynthetic active radiation induce formation of mycosporine-like amino acids in the marine macroalga Chondrus crispus (Rhodophyta). Planta 205: 257-262.

KARSTEN U, T SAWALL, D HANELT, K BISCHOF, FL FIGUEROA, A FLORES-MOYA \& C WIENCKE (1998b) An inventory of UV-absorbing mycosporine-like amino acids in macroalgae from polar to warm-temperate regions. Botanica Marina 41: 443-453.

KARSTEN U, K BISCHOF, D HANELT, H TÜG \& C WIENCKE (1999) The effect of ultraviolet radiation on photosynthesis and ultravioletabsorbing substances in the endemic Arctic macroalga Devaleraea ramentacea (Rhodophyta). Physiologia Plantarum 105: 58-66.

KARSTEN U, A DUMMERMUTH, K HOYER \& C WIENCKE (2003) Interactive effects of ultraviolet radiation and salinity on the ecophysiology of two Arctic red algae from shallow waters. Polar Biology 26: $249-258$.

KERR JB \& CT MCELROY (1993) Evidence for large upward trends of ultraviolet-B radiation linked to ozone depletion. Science 262: 1032-1034.

KINZIE RAIII (1993) Effects of ambient levels of solar ultraviolet radiation on zooxanthellae and photosynthesis of the reef coral Montipora verrucosa. Marine Biology 116: 319-327.

KLISCH M \& DP HÄDER (2000) Mycosporine-like amino acids in the marine dinoflagellate
Gyrodinium dorsum: induction by ultraviolet irradiation. Journal of Photochemistry and Photobiology B 55: 178-182.

KORBEE-PEINADO N (2004) Fotorregulación y efecto del nitrógeno inorgánico en la acumulación de aminoácidos tipo micosporina en algas rojas. Tesis Doctoral, Servicio de Publicaciones, Universidad de Málaga, Málaga, España. 279 pp.

KORBEE PEINADO N, RT ABDALA-DÍAZ, FL FIGUEROA \& EW HELBLING (2004) Ammonium and UV radiation stimulate the accumulation of mycosporine-like amino acids in Porphyra columbina (Rhodophyta) from Patagonia, Argentina. Journal of Phycology 40: 248-259.

KORBEE N, P HUOVINEN, FL FIGUEROA, J AGUILERA, U KARSTEN (2005) Availability of ammonium influences the photosynthesis and the accumulation of MAAs in two Porphyra species (Bangiales, Rhodophyta) from different latitudes. Marine Biology 146: 645-654.

LEACH CM (1965) Ultraviolet-absorbing substances associated with light-induced sporulation in fungi. Canadian Journal of Botany 43: 185-200.

LEAVITT PR, RD VINEBROOKE, DB DONALD, JP SMOL, DW SCHINDLER (1997) Past ultraviolet radiation environments in lakes derived from fossil pigments. Nature 388: 457-459.

LESSER MP (1996) Elevated temperature and ultraviolet radiation cause oxidative stress and inhibit photosynthesis in symbiotic dinoflagellates. Limnology and Oceanography 41: 271-283.

LESSER MP (2000) Depth-dependent photoacclimatization to solar ultraviolet radiation in the Caribbean coral Montastraea faveolata. Marine Ecology Progress Series 192: 137-151.

LESSER MP, VM WEIS, MR PATTERSON \& PL JOKIEL (1994) Effects of water motion on carbon delivery and productivity in the reef coral, Pocillopora damicornis (Linnaeus): diffusion barriers, inorganic carbon limitation, and biochemical plasticity. Journal of Experimental Marine Biology and Ecology 178: 153-179.

LITCHMAN E, PJ NEALE \& AT BANASZAK (2002) Increased sensitivity to ultraviolet radiation in nitrogen-limited dinoflagellates: photoprotection and repair. Limnology and Oceanography 47: 8694

LOGAN A, K HALCROW \& T TOMASCIK (1990) UV excitation-fluorescence in polyp tissue of certain scleractinian corals from Barbados and Bermuda. Bulletin of Marine Science 46: 807-813.

MARAGOS JE (1972) A study of the ecology of Hawaiian reef corals. Tesis Doctoral, Oceanography Department, Universidad de Hawaii, Honolulu, Hawaii, USA. 290 pp.

MISONOU T, J SAITOH, S OSHIBA, Y TOKITOMO, M MAEGAWA,Y INOUE, H HORI \& T SAKURAI (2003) UV-absorbing substance in the red alga Porphyra yezoensis (Bangiales, Rhodophyta) block thymine photodimer production. Marine Biotechnology 5: 194-200.

MITCHELL DL \& D KARENTZ (1993) The induction and repair of DNA photodamage in the environment. En: Young AR, LO Björn, J Moan \& W Nultsch (eds) Environmental UV photobiology: 345-377. Plenum Press, New York, New York, USA.

MOISAN TA \& BG MITCHELL (2001) UV absorption by mycosporine-like amino acids in Phaeocystis Antarctica Karsten induced by photosynthetically available radiation. Marine Biology 138: 217-227. 
MOLINA X \& V MONTECINO (1996) Acclimation to UV irradiance in Gracilaria chilensis bird, McLachlan \& Oliveira (Gigartinales, Rhodophyta). Hydrobiologia 326/327: 415-420.

MUSZYNSKI FZ, A BRUCKNER, RA ARMSTRONG, JM MORELL \& JE CORREDOR (1998) Within-colony variations of UV absorption in a reef building coral. Bulletin of Marine Science 63: 589-594.

NEALE PJ, AT BANASZAK \& CR JARRIEL (1998) Ultraviolet sunscreens in Gymnodinium sanguineum (Dinophyceae): mycosporine-like amino acids protect against inhibition of photosynthesis. Journal of Phycology 34: 928-938.

NEWMAN ST, WC DUNLAP, S NICOL \& D RITZ (2000) Antarctic krill (Euphausia superba) acquires a UV-absorbing mycosporine-like amino acid from dietary algae. Journal of Experimental Marine Biology and Ecology 255: 93-110.

OREN A (1997) Mycosporine-like amino acids as osmotic solutes in a community of halophilic cyanobacteria. Geomicrobiology Journal 14: 231-240.

PORTWICH A \& F GARCÍA-PICHEL (1999) Ultraviolet and osmotic stresses induce and regulate the synthesis of mycosporines in the cyanobacterium Chlorogloeopsis PCC 6912. Archives of Microbiology 172: 187-192.

PORTWICH A \& F GARCÍA-PICHEL (2003) Biosynthetic pathway of mycosporines (mycosporine-like amino acids) in the cyanobacterium Chlorogloeopsis sp. Strain PCC 6912. Phycologia 42: 384-392.

POST A \& AWD LARKUM (1993) UV-absorbing pigments, photosynthesis and UV-exposure in Antarctica: comparison of terrestrial and marine algae. Aquatic Botany 45: 231-243.

PRICE JH \& HS FORREST (1969) $310 \mathrm{~nm}$ absorbance in Physalia physalis: distribution of the absorbance and isolation of a $310 \mathrm{~nm}$ absorbing compound. Comparative Biochemistry Physiology 30: 879-888.

QUESADA A, WF VINCENT \& DRS LEAN (1999) Community and pigment structure of Artic cyanobacterial assemblages: the occurrence and distribution of UV-absorbing compounds. FEMS Microbiology Ecology 28: 315-323.

RIEGGER L \& D ROBINSON (1997) Photoinduction of UV-absorbing compounds in Antarctic diatoms and Phaeocystis antarctica. Marine Ecology Progress Series 160: 13-25

SCHMID D, C SCHUERCH \& F ZUELLI (2004) Harmless, natural cosmetic skin treatment compositions, for protecting against UV-A induced lipid oxidation and premature aging, containing mycosporine-like aminoacids. Patent number: EP1473028-A1, Zurich, Suiza. 13 pp.

SHIBATA K (1969) Pigments and a UV-absorbing substance in corals and a blue-green alga living in the Great Barrier Reef. Plant \& Cell Physiology 10: 325-335

SHICK JM \& WC DUNLAP (2002) Mycosporine-like amino acids and related gadusols: Biosynthesis, accumulation and UV-protective functions in aquatic organisms. Annual Review of Physiology 64: 223-262.

SHICK JM, MP LESSER \& WR STOCHAJ (1991) Ultraviolet radiation and photooxidative stress in zooxanthellate Anthozoa: the sea anemone Phyllodiscus semoni and the octocoral Clavularia sp. Symbiosis 10: 145-173.

SHICK JM, SD ROMAINE-LIOUD, C FERRIER-PAGES \& JP GATTUSO (1999) Ultraviolet-B radiation stimulates shikimate pathway-dependent accumulation of mycosporine-like amino acids in the coral Stylophora pistillata despite decreases in its population of symbiotic dinoflagellates. Limnology and Oceanography 44: 1667-1682.

SHICK JM, WC DUNLAP \& GR BUETTNER (2000) Ultraviolet (UV) protection in marine organisms II. Biosynthesis, accumulation, and sunscreening function of mycosporine-like amino acids. En: Yoshikawa S, S Toyokuni, Y Yamamoto \& Y Naito (eds) Free radicals in chemistry, biology and medicine: 215-228. OICA International, London, United Kingdom.

SHICK JM, WC DUNLAP, JS PEARSE \& VB PEARSE (2002) Mycosporine-like amino acid content in four species of sea anemones in the genus Anthopleura reflects phylogenetic but not environmental or symbiotic relationships. Biological Bulletin 203: 315-330.

SINHA RP, M KLISCH, A GRÖNIGER \& DP HÄDER (2000) Mycosporine-like amino acids in the marine red alga Gracilaria cornea-effects of UV and heat. Environmental and Experimental Botany 43: 33-43.

SINHA RP, NK AMBASHT, JP SINHA \& DP HÄDER (2003) Wavelength-dependent induction of a mycosporine-like amino acid in a rice-field cyanobacterium, Nostoc commune: role of inhibitors and salt stress. Photochemical \& Photobiological Science 2: 171-176.

SIVALINGAM PM, T IKAWA \& K NISIZAWA (1976a) Isolation and physico-chemical properties of a substance 334 from the red alga, Porphyra yezoensis Ueda. Botanica Marina 19: 1-7.

SIVALINGAM PM, T IKAWA \& K NISIZAWA (1976b) Physiological roles of a substance 334 in algae. Botanica Marina 19: 9-21

STOCHAJ WR, WC DUNLAP \& JM SHICK (1994) Two new UV-absorbing mycosporine-like amino acids from the sea anemone Anthopleura elegantissima and the effects of spectral irradiance on chemical composition and content. Marine Biology 118: 149156.

SUH HJ, HW LEE \& J JUNG (2003) Mycosporine glycine protects biological systems against photodynamic damage by quenching singlet oxygen with a high efficiency. Photochemistry and Photobiology 78: 109-113.

TAKANO S, D UEMURA \& Y HIRATA (1978a) Isolation and structure of a new amino acid, palythine, from the zoanthid Palythoa tuberculosa. Tetrahedron Letters 26: 2299-2300.

TAKANO S, D UEMURA \& Y HIRATA (1978b) Isolation and structure of two new amino acids, palythinol and palythene, from the zoanthid Palythoa tuberculosa. Tetrahedron Letters 26: 4909-4912.

TALARICO L \& G MARANZANA (2000) Light and adaptive responses in red macroalgae; an overview. Journal of Photochemistry Photobiology B: Biology $56: 1-11$

TANDEAU DE MARSAC N \& J HOUMARDD (1993) Adaptation of cyanobacteria to environmental stimuli: new steps towards molecular mechanisms. FEMS Microbiology Reviews 104: 119-190.

TEAI T, JH DROLLET, JP BIANCHINI, A CAMBON \& PMV MARTIN (1997) Widespread occurrence of mycosporine-like amino acid compounds in scleractinians from French Polynesia. Coral Reefs 16: 169-176.

TSUJINO I \& T SAITO (1961) Studies on the compounds specific for each group of marine alga. I. Presence 
of characteristic ultraviolet absorbing material in Rhodophyceae. Bulletin of the Faculty of Fish, Hokkaido University (Japón) 12: 49-58.

TSUJINO I, K YABE \& I SEKEKAWA (1980) Isolation and structure of a new amino acid, shinorine, from the red alga Chondrus yendoi Yamada et Mikami. Botanica Marina 23: 65-68.

VAN DE POLL WH, A EGGERT, AGJ BUMA, AM BREEMAN (2001) Effects of UV-B-induced DNA damage and photoinhibition on growth of temperate marine red macrophytes: habitat-related differences in UV-B tolerance. Journal of Phycology 37: 30-37.

VERNET M \& K WHITEHEAD (1996) Release of ultraviolet-absorbing compounds by the red-tide dinoflagellate Lingulodinium polyedrum. Marine Biology 127: 35-44.

VERNET M, A NEORI \& FT HAXO (1989) Spectral properties and photosynthetic action in red-tide populations of Prorocentrum micans and Gonyaulax polyhedra. Marine Biology 103: 365-371.

VILLAFAÑE V, C SUNFBÄCK, FL FIGUEROA \& WE HELBLING (2003) Photosynthesis in the aquatic environment as affected by ultraviolet radiation. En: Helbling EW \& H Zagarese (eds) UV effects in aquatic and Ecosystems: 357-397. European Society for Photobiology, Comprehensive Series in Photosciences, The Royal Society of Chemistry, Cambridge, United Kingdom.

VINCENT WF \& PJ NEALE (2000) Mechanisms of UV damage to aquatic organisms. En: de Mora SJ, S Demers \& M Vernet (eds) The effects of UV radiation in the marine environment: 149-176. Cambridge University Press, New York, USA.

VIÑEGLA B (2000) Efecto de la radiación ultravioleta sobre actividades enzimáticas relacionadas con el metabolismo del carbono y nitrógeno en macroalgas y fanerógamas marinas. Tesis Doctoral, Facultad de Ciencias, Universidad de Málaga, Málaga, España. $404 \mathrm{pp}$.

WÄNGBERG SA, A PERSSON \& B KARLSON (1997) Effects of UV-B radiation on synthesis of mycosporine-like amino acid and growth in Heterocapsa triquetra (Dinophyceae). Journal of Photochemistry and Photobiology 37: 141-146.

WHITEHEAD K \& M VERNET (2000) Influence of mycosporine-like amino acids (MAAs) on UV absorption by particulate and dissolved organic matter in La Jolla Bay. Limnology and Oceanography 45: 1788-1796.

WIENCKE C, I GÓMEZ, H PAKKER, A FLORESMOYA, M ALTAMIRANO, D HANELT, K BISCHOF \& FL FIGUEROA (2000) Impact of UV radiation on viability, photosynthetic characteristics and DNA on algal zoospores: implications for depth zonation. Marine Ecology Progress Series 197: 217-219.

WOOD WF (1989) Photoadaptive responses of the tropical red alga Eucheuma striatum Schmitz (Gigartinales) to ultraviolet radiation. Aquatic Botany 33: 41-51.

WU WON JJ, JA RIDEOUT \& BE CHALKER (1995) Isolation and structure of a novel mycosporine-like amino acid from the reef-building corals Pocillopora damicornis and Stylophora pistillata. Tetrahedron Letters 36: 5255-5256.

WU WON JJ, BE CHALKER \& JA RIDEOUT (1997) Two new UV-absorbing compounds from Stylophora pistillata: sulfate esters of mycosporine-like amino acids. Tetrahedron Letters 38: 2525-2526.

YAKOVLEVA IM \& EA TITLYANOV (2001) Effect of high visible and UV irradiance on subtidal Chondrus crispus: stress, photoinhibition and protective mechanisms. Aquatic Botany 71: 47-61. 